\title{
FMRP Expression Levels in Mouse Central Nervous System Neurons Determine Behavioral Phenotype
}

\author{
Jason Arsenault, Shervin Gholizadeh, ${ }^{1, \dagger}$ Yosuke Niibori, ${ }^{1, \dagger}$ Laura K. Pacey, ${ }^{1}$ Sebok K. Halder, \\ Enea Koxhioni, ${ }^{1}$ Ayumu Konno, Hirokazu Hirai, ${ }^{2}$ and David R. Hampson ${ }^{1,3, *}$ \\ ${ }^{1}$ Department of Pharmaceutical Sciences, Leslie Dan Faculty of Pharmacy, University of Toronto, Toronto, Ontario, Canada; ${ }^{2}$ Department of Neurophysiology \\ and Neural Repair, Gunma University Graduate School of Medicine, Maebashi, Japan; and ${ }^{3}$ Department of Pharmacology, Faculty of Medicine, \\ University of Toronto, Toronto, Ontario, Canada. \\ $\dagger$ These authors contributed equally to this study.
}

Fragile X mental retardation protein (FMRP) is absent or highly reduced in Fragile X Syndrome, a genetic disorder causing cognitive impairment and autistic behaviors. Previous proof-of-principle studies have demonstrated that restoring FMRP in the brain using viral vectors can improve pathological abnormalities in mouse models of fragile X. However, unlike small molecule drugs where the dose can readily be adjusted during treatment, viral vector-based biological therapeutic drugs present challenges in terms of achieving optimal dosing and expression levels. The objective of this study was to investigate the consequences of expressing varying levels of FMRP selectively in neurons of Fmr1 knockout and wild-type (WT) mice. A wide range of neuronal FMRP transgene levels was achieved in individual mice after intra-cerebroventricular administration of adeno-associated viral vectors coding for FMRP. In all treated knockout mice, prominent FMRP transgene expression was observed in forebrain structures, whereas lower levels were present in more caudal regions of the brain. Reduced levels of the synaptic protein PSD-95, elevated levels of the transcriptional modulator MeCP2, and abnormal motor activity, anxiety, and acoustic startle responses in Fmr1 knockout mice were fully or partially rescued after expression of FMRP at about 35-115\% of WT expression, depending on the brain region examined. In the WT mouse, moderate FMRP over-expression of up to about twofold had little or no effect on PSD-95 and MeCP2 levels or on behavioral endophenotypes. In contrast, excessive over-expression in the Fmr1 knockout mouse forebrain (approximately 2.5-6-fold over WT) induced pathological motor hyperactivity and suppressed the startle response relative to WT mice. These results delineate a range of FMRP expression levels in the central nervous system that confer phenotypic improvement in fragile X mice. Collectively, these findings are pertinent to the development of long-term curative gene therapy strategies for treating Fragile X Syndrome and other neurodevelopmental disorders.

Keywords: adeno-associated virus, autism, Fmr1, fragile X syndrome, MeCP2, Rett syndrome

\section{INTRODUCTION}

FRAGILE X SYNDROME (FXS) is a neurodevelopmental disorder caused by an expanded CGG repeat in the $5^{\prime}$ untranslated region of the X-linked FMR1 gene, resulting in elimination or drastic reduction of the encoded fragile $\mathrm{X}$ mental retardation protein (FMRP). FMRP functions as an mRNAbinding protein to regulate the expression of hundreds of mRNAs in the brain and other organs. Most but not all of the symptoms of the disorder stem from deficient FMRP in the central nervous system (CNS). Neurological symptoms include cognitive impairment, hyperactivity, stereotyped behaviors, reduced and/or abnormal social interactions, and epileptic seizures. ${ }^{1}$ Approximately $30 \%$ of patients with FSX meet the clinical cri-

* Correspondence: Dr. David R. Hampson, Department of Pharmaceutical Sciences, Leslie Dan Faculty of Pharmacy, University of Toronto, 144 College Street, Toronto, ON, Canada M5S3M2. E-mail: d.hampson@utoronto.ca

(C) Jason Arsenault, et al., 2016; Published by Mary Ann Liebert, Inc. This Open Access article is distributed under the terms of the Creative Commons Attribution Noncommercial License (http://creativecommons.org/licenses/by-nc/4.0/), which permits any noncommercial use, distribution, and reproduction in any medium, provided the original author(s) and the source are credited. 
teria for autism, and as with autism, FXS is a lifelong disorder where the behavioral symptoms appear around 2 years of age and remain throughout life.

There are currently no drugs available to treat FXS directly. Instead, antipsychotics, antidepressants, stimulants, and anti-epileptic drugs are used to suppress symptoms in children and adults. ${ }^{2}$ Newer second-generation drugs acting at metabotropic glutamate and $\mathrm{GABA}_{\mathrm{B}}$ receptors rely on single molecular targets, and were expected to be more effective in alleviating selected symptoms. ${ }^{1,3,4}$ However, to date, none of the small molecule drugs tested have shown convincing efficacy in Phase II clinical trials for FXS or autism. ${ }^{5,6}$ Given that FMRP regulates the stability and translation of hundreds of mRNAs in the CNS, ${ }^{7,8}$ these narrowly defined molecular treatment strategies have not addressed the core underlying issue: the absence or reduction of FMRP in the brain.

In principle, FXS should be amenable to correction following treatment with viral vectors coding for FMRP. Key experimental parameters requiring consideration in viral vector-mediated gene therapy strategies for CNS disorders include the age at time of treatment and the route of administration. ${ }^{9,10} \mathrm{In}$ the case of FXS, FMRP is distributed in neurons throughout the brain at all ages. ${ }^{11}$ Therefore, a gene therapy approach for treating FXS would likely necessitate widespread distribution of the transgene throughout the brain at appropriate levels and over a protracted time frame. The adeno-associated viral (AAV) vectors now in use are well-suited for meeting these requirements, ${ }^{12-14}$ and in fact, several studies have demonstrated the efficacy of AAV-based therapies in several mouse models of neurodevelopment disorders, including Angelman Syndrome, ${ }^{15}$ Rett Syndrome, ${ }^{16-18}$ and tuberous sclerosis (TSC). ${ }^{19}$ In the context of FXS, early work by Zeier et al. ${ }^{20}$ showed that abnormal (enhanced) hippocampal long-term depression could be rectified after direct injection of an AAV serotype 5 vector coding for FMRP into 5-week-old Fmr 1 knockout (KO) mouse hippocampus. Previous work from our laboratory demonstrated the utility of early postnatal intracerebroventricular (i.c.v.) administration of an AAV serotype 9 vector coding for FMRP in correcting selected abnormal behaviors, including elevated repetitive and reduced social dominance behaviors in Fmr1 KO mice. ${ }^{21}$

One of several critical parameters that must be considered in treating genetic disorders is the level of expression of the recombinant transgene. Too little expression may be insufficient to restore the normal function of the protein, while overexpression could induce pathology. Biologically based therapeutic agents in general, and viral vectors in particular, differ from small molecule drugs in terms of biodistribution, pharmacokinetics, and dose adjustability during treatment. ${ }^{22}$ Unlike small molecule drugs where dosing is done on a repeated basis and opportunities exist for adjusting the dose up or down, viral vector-based drugs confer long-term transgene expression in the CNS and are typically administered only once or a few times. ${ }^{23}$ Thus, with viral vector-based CNS therapies, it becomes important to optimize the dose upon initiation of therapy. The goal of the present study was to examine the consequences of inducing varying amounts of FMRP expression in neurons of the Fmr1 KO mouse. The results delineate a range of FMRP expression levels in the CNS that confer phenotypic improvement in fragile $\mathrm{X}$ mice, and conversely, an upper limit of expression above which is associated with pathological features.

\section{EXPERIMENTAL PROCEDURES}

\section{Animals and AAV vectors}

C57/BL6J wild-type (WT) mice and Fmr1 KO mice on the C57/BL/6J strain were used in this study. Single-stranded AAV vectors containing inverted terminal repeat DNA sequences from the genome of AAV2, the capsid proteins from AAV9, the human or rat synapsin-1 (Syn) promoter, the coding region of Mus musculus FMRP (isoform 1), a downstream Woodchuck Hepatitis posttranscriptional regulatory element (WPRE), and a poly adenylation site were used. The vector designated here as "Syn-FMRP" containing these domains was previously described. ${ }^{21}$ The vector designated here as "Syn-mCMV-FMRP" also contains these domains, and a minimal cytomegalovirus (mCMV) sequence ${ }^{24}$ was inserted at the $3^{\prime}$ end of the synapsin promoter, upstream of the FMRP transgene (see Fig. 1). Virus production was carried out at the University of Pennsylvania Vector Core Facility; purified AAVs were used at a concentration range from $0.5-1.0 \times 10^{13}$ genomes $/ \mathrm{mL}$ in sterile phosphate-buffered saline (PBS) and stored at $-80^{\circ} \mathrm{C}$. A third single-stranded AAV vector of the same serotype containing the CMV promoter without the transgene (AAV null) was prepared in the same manner and used as a negative control.

\section{Vector injections}

A single bilateral i.c.v. injection of AAV vector was administered to WT and Fmr1 KO mice as described previously ${ }^{9,21}$ with some modifications. Briefly, mouse pups between postnatal days (PND) 0 and 2 were immobilized by cryo-anesthesia for 2 min and then gently grasped by the skin behind 


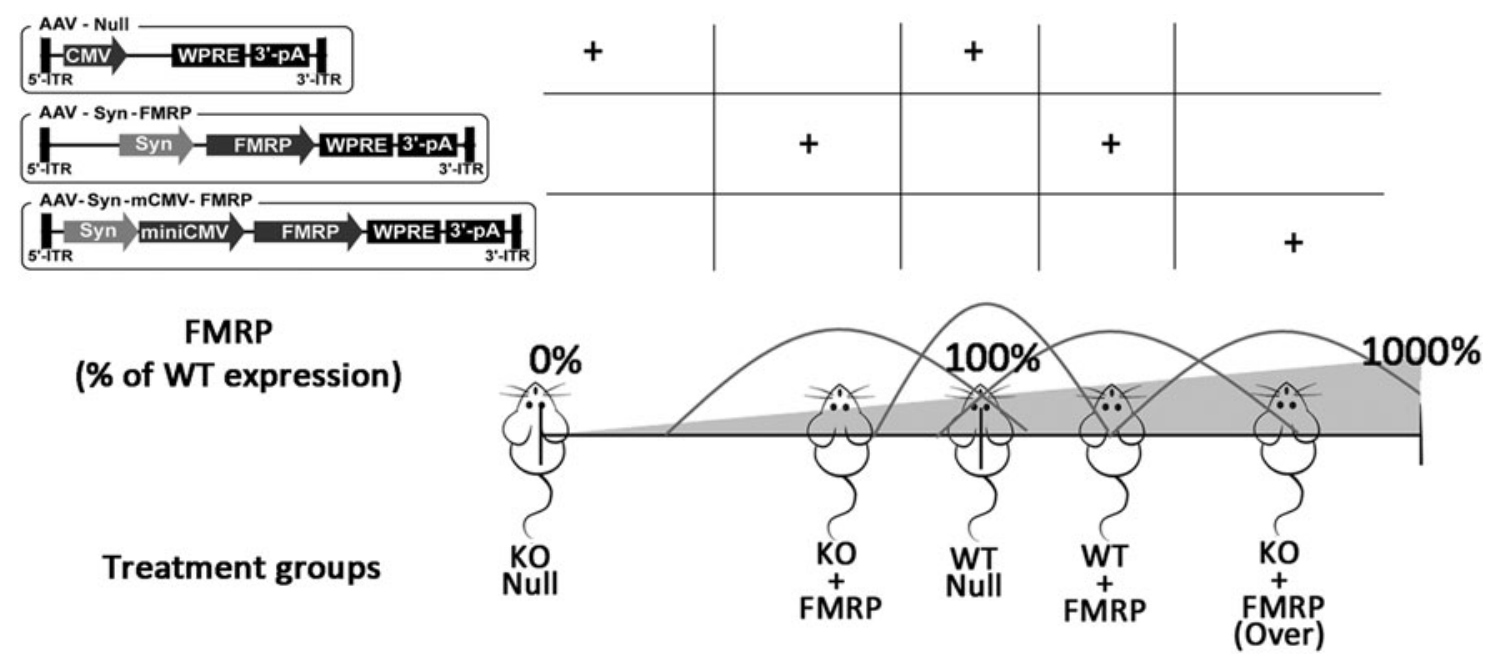

Figure 1. AAV vectors and treatment groups. Top left, schematic of the three AAV vectors injected into wild-type (WT) and Fmr1 knockout (KO) mice. Bottom: the five treatment groups presented in order (left to right) of fragile X mental retardation protein (FMRP) expression levels: KO-null, KO + FMRP, WT null, WT + FMRP, and KO + FMRP (over-expression). + symbols above indicate the vector used in that treatment group.

the head and placed over a fiber-optic light to illuminate the transverse and midline sutures that were used as guides for the injections. A 30-gauge needle attached to a $1 \mathrm{~mL}$ Hamilton syringe (Hamilton) through long fluoropolymer tubing (internal diameter of $0.5 \mathrm{~mm}$; Western Analytical) was used for injections. The needle was inserted at a depth of $2 \mathrm{~mm}$ perpendicular to the skull surface, $0.25 \mathrm{~mm}$ lateral to the sagittal suture, and 0.50 $0.75 \mathrm{~mm}$ rostral to the neonatal coronary suture. A volume range of $0.75-1.0 \mu \mathrm{L}$ of the AAV vectors was injected using a syringe pump at the rate of $1 \mu \mathrm{L} /$ min into each lateral ventricle. After the injection, the needle was maintained for $1 \mathrm{~min}$ in the inserted position and then slowly retracted to prevent backflow. WT and Fmr1 KO mice injected with the AAV null vector served as the control groups.

\section{Immunocytochemistry and confocal microscopy}

Female mouse brains were used for immunocytochemistry. At $70 \pm 3$ days post injection, the mice were anesthetized with ketamine and xylazine and transcardially perfused with PBS followed by $4 \%$ paraformaldehyde in PBS ( $\mathrm{pH}$ 7.4). Serial coronal or sagittal sections were cut at a thickness of $25 \mu \mathrm{m}$ using a cryostat (Leica 9 Microsystems) as previously described. ${ }^{11,21}$ Free-floating sections were washed with PBS and antigen retrieval was performed as described previously. ${ }^{25}$ Mouse monoclonal anti-FMRP $\left(5 \mathrm{c} 2 ;{ }^{26}\right.$ was used with either rabbit anti-NeuN (1:1,000; Abcam) to immunolabel neurons, or rabbit anti-S100 $\beta$ (1:1,000; Abcam) to label astrocytes. After overnight incubation, sections were washed five times with PBS for $10 \mathrm{~min}$ and incubated with secondary antibodies (goat anti-mouse Alexa Fluor 488 and goat antirabbit Alexa Fluor 594; 1:1,000 dilution; Jackson ImmunoResearch Laboratories, Inc.) diluted in PBS containing 5\% goat serum. DAPI (SigmaAldrich) was used to label nuclei. The images were captured using a laser scanning confocal microscope (LSM 700; Carl Zeiss) at 10, 20, 40, or $63 \times$ magnifications and analyzed using the Zen software (Carl Zeiss). The images for sagittal sections were captured using a Zeiss Mirax slide scanner at $20 \times$ magnification.

For a comparative analysis of the transduction pattern in the brain, a semi-quantitative scoring system was used to analyze the transduction of the FMRP transgene in different brain regions of $F m r 1$ $\mathrm{KO}$ mice injected with AAV-Syn-FMRP or AAVSyn-mCMV-FMRP. Scoring was done by classifying the number of transduced cells into four categories: regions with no detectable FMRP expression (-), regions with 0-100 FMRP positive cells $/ \mathrm{mm}^{2}(+)$, regions with 100-200 FMRP-positive cells (++), regions with 200-300 FMRP-positive cells $(++)$, and regions with $>300$ FMRP-positive cells $(++1+)$. The cingulate cortex was used to calculate the total number of FMRP-positive cells in each KO + FMRP groups, the percentage of FMRP-positive cells that co-localized with the neuronal marker NeuN, and the percentage of FMRP-positive cells were counted.

\section{Quantitative Western blotting}

Male mouse brains were used for Western blotting. Samples of the cerebellum, hippocampus, and striatum were collected and stored at $-80^{\circ} \mathrm{C}$. The caudal segment of the cerebral cortex, herein re- 
ferred as "cortex," which included the retrospenial area, anterior cingulate area, visual cortex, and the posterior somatomotor area, was also harvested and stored at $-80^{\circ} \mathrm{C}$. The rostral segment of the cerebral cortex, herein referred as the "frontal cortex," which comprised the anterior somatomotor, prelimbic, orbital, and inferior limbic areas, was also harvested (see Fig. 2 for schematic representation of the regions investigated; mouse brain anatomical regions and nomenclature conform to the Allen Mouse Brain Reference Atlas).

The samples were homogenized in ice-cold TrisSDS buffer (50 mM Tris, $1 \%$ SDS, pH 7.4) containing complete protease inhibitor cocktail (Roche). Protein $(10 \mu \mathrm{g})$ was loaded per well and resolved on $10 \%$ acrylamide gels. Following SDS-PAGE electrophoresis, the proteins were transferred onto nitrocellulose membranes and incubated overnight with primary antibodies, including mouse anti-FMRP $5 \mathrm{C} 2$ at $1: 1,000$, rabbit monoclonal anti-MeCP2 at 1:1,000 (D4F3), mouse monoclonal anti-PSD-95 at 1:250,000 (75-028; NeuroMab) dilutions, and mouse monoclonal anti-GAPDH (GAPDH-71.1; SigmaAldrich) at a 1:40,000 dilution, which was used as a loading control. After washing, the membranes were incubated with HRP-conjugated goat antimouse or anti-rabbit secondary antibodies (Jackson ImmunoResearch Laboratories, Inc.), then SuperSignal West Pico Chemiluminescent Substrate (Thermo Scientific) was added to the membranes,
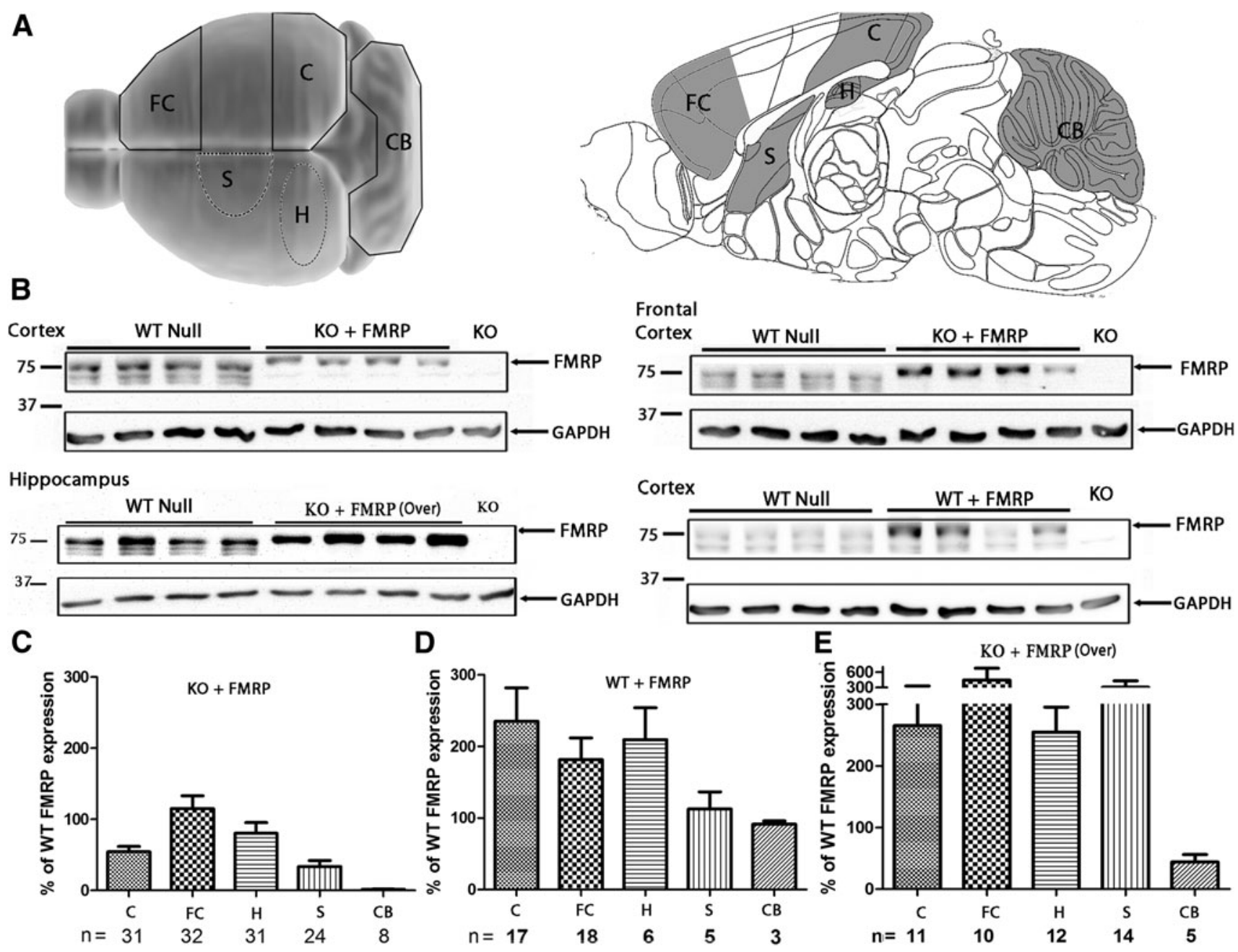

Figure 2. Brain FMRP transgene expression levels based on Western blotting. (A) Schematic representation of the brain regions investigated (top view, left sagittal view, right): cortex excluding frontal cortex (C), frontal cortex (FC), cerebellum (CB), striatum (S), and hippocampus (H). (B) Representative Western blots of FMRP expression in the mouse brain. FMRP transgene expression in Fmr1 KO mice injected with Syn-FMRP are shown for the cortex (top left) and frontal cortex (top right). The lower left panel shows the FMRP expression levels in the hippocampus of Fmr1 K0 mice injected with Syn-mCMV-FMRP. The lower right panel shows the FMRP expression levels in the cortex of WT mice injected with Syn-FMRP. (C) Summary of quantification of FMRP in different brain regions of Fmr1 KO mice injected with Syn-FMRP and (D) WT injected with Syn-FMRP. (E) Quantification of FMRP expression in Fmr1 KO mice injected with Syn-mCMV-FMRP. Protein levels were normalized to WT null expression and further corrected for protein loading based on GAPDH expression. The results are presented as the means \pm standard error of the mean (SEM). $n$ indicates the number of individual mice analyzed in each condition. 
and the bands were revealed using an Alpha Innotec Fluorochem gel imager (Protein Simple). The AlphaEase SA software was used to analyze the images. Quantification of protein expression was normalized using GAPDH expression; $100 \%$ levels were based on the average of the WT null group's protein expression. Results are presented as means \pm standard error of the mean (SEM). Statistical significance was determined using GraphPad Prism v5 by using one-way analysis of variance and Tukey's multiple comparison test.

\section{Immunoprecipitation and quantitative reverse transcription polymerase chain reaction}

Immunoprecipitation followed by quantitative reverse transcription polymerase chain reaction (RT-PCR) was performed using a modification of the method described by Pacey et al. ${ }^{30}$ Briefly, whole mouse forebrains from naive PND $60 \mathrm{WT}$ mice or HEK-293 cells were pooled and homogenized with $3 \mathrm{~mL}$ of IP buffer $(10 \mathrm{mM}$ of HEPES, $200 \mathrm{mM}$ of $\mathrm{NaCl}, 30 \mathrm{mM}$ of EDTA, $0.5 \%$ Triton $\mathrm{X}-100, \mathrm{pH} 7.4$, with the addition of Ribolock RNase inhibitor; Thermo Scientific) and $1 \times$ complete protease inhibitor cocktail (Roche). Lysates were centrifuged at $3,000 \mathrm{~g}$ for $10 \mathrm{~min}$. and pellets were washed once with $2 \mathrm{~mL}$ of IP buffer. Supernatants were combined, and the $\mathrm{NaCl}$ concentration was adjusted to $400 \mathrm{mM}$ and centrifuged again at $70,000 \mathrm{~g}$ for $30 \mathrm{~min}$, and total protein concentrations were adjusted to $4 \mathrm{mg} / \mathrm{mL}$. The solution was pre-cleared using protein A magnabeads ${ }^{\circledR}$ (Genscript) for $1 \mathrm{~h}$ at $4^{\circ} \mathrm{C}$. The supernatant was then incubated with the anti-FMRP 5c2 antibody for $2 \mathrm{~h}$ at $4^{\circ} \mathrm{C}$ under constant agitation. Protein $\mathrm{A}$ beads blocked with $0.1 \mathrm{mg} / \mathrm{mL}$ of bovine serum albumin and $0.1 \mathrm{mg} / \mathrm{mL}$ of yeast tRNA (Thermo Scientific) were added to the solution and incubated overnight. The supernatant was removed, and the beads were washed four times with ice-cold IP buffer. The beads were re-suspended in TRIzol ${ }^{\circledR}$ reagent (Thermo Scientific), and RNA isolation was performed following the manufacturer's instructions. RNA was re-suspended in RNAse free $\mathrm{H}_{2} \mathrm{O}$ and reverse transcribed using random nanomers (Sigma-Aldrich) and the superscript II reverse transcriptase (Thermo Scientific) according to the manufacturer's protocol. Quantitative RTPCR was performed with an ABI Prism 7900 HT (Thermo Scientific) using a $\mathrm{SyBr}$ green detection system (Sigma-Aldrich), and samples were evaluated for the housekeeping gene GAPDH (forward 5'-CGGTGCTGAGTATGTCGTGGAGTC; reverse 5'-CTTTTGGCTCCACCCTTCAAGTG) and MeC P2 (forward 5'-CGTGACCGGGGACCTATGTA; reverse 5'-AAGCTTTCGTGTCCAACC), as well as PSD-95 (forward 5'-AAGCCCCAGGATATGTGA ACGG; reverse 5'-AGCCCAGACCTGAGTTACCC CTT) for the forebrain sample or HEK-293 cell homogenate, using the standard curve method. mRNA enrichment was calculated as the ratio of mRNA in the FMRP-immunoprecipitate divided by the total mRNA isolate and normalized to the GAPDH levels. Results are presented as means \pm SEM. Statistical significance was determined with GraphPad Prism v5 by using a two-tailed Student's $t$-test.

\section{Behavioral analyses}

All behavioral tests were conducted on age- and sex-matched animals. All mice were naive to each test, tested only once in each procedure, and the experimenters were blinded to the treatment group. Behavioral testing started on PND $55 \pm 2$, and each test was performed at least 2 days after the previous test in the order presented below. Each test was always performed either in the morning between 9:00a.m. and 12:00p.m., or in the afternoon between 12:00p.m. and 4:00p.m. The results are presented as means \pm SEM.

\section{Locomotor activity measurements}

At PND 55 \pm 2 , locomotor activity was assessed using an automated open field locomotor monitor system (Accuscan Images). Mice were acclimated to the testing room for $5 \mathrm{~min}$ and then placed in the open field and monitored for $20 \mathrm{~min}$ in low light conditions. The total horizontal distance covered, horizontal activity ( $x$-axis beam breaks), ambulatory activity (total stationary and mobile movements), the number of rest episodes (incidence), and the total rest time were recorded using Fusion software and compared between groups.

\section{Elevated plus maze}

At PND $62 \pm 2$, the elevated plus maze (EPM) was used to monitor open field avoidance, exploration, and general anxiety. Each arm of the apparatus (San Diego Instruments, SR-Lab System) was $5 \mathrm{~cm} \times 30.5 \mathrm{~cm}$, and the closed arms contained a $15 \mathrm{~cm}$ high wall. The platform stood $40 \mathrm{~cm}$ above the table. The mice were allowed to acclimatize to the room for $15 \mathrm{~min}$ and then placed in the central pedestal and monitored for $5 \mathrm{~min}$ while the experimenter sat $2 \mathrm{~m}$ away, directly in line with the closed arms. Mouse activity was visually recorded from a bird's eye view using a camera mounted on the ceiling. An entry was defined as having all four paws completely outside the central segment. The time spent in the open and closed arms and the number of arm entries were compared between groups. 


\section{Prepulse inhibition and startle response}

At PND 67 \pm 2 , acoustic startle responses and prepulse inhibitions (PPI) were measured to monitor sensorimotor gating. The chambers, comprising a sound attenuation chamber and a cylindrical tube inside of which the animal was placed during testing, were obtained from San Diego Instruments. Trials and settings were programmed as previously described. ${ }^{27,28}$ Briefly, each session consisted of 48 trials divided into six blocks of eight pseudo-randomly presented conditions. Each block contained one "no stimulus" trial to measure baseline movement, a startle burst only ( $120 \mathrm{~dB}$ lasting $40 \mathrm{~ms})$, three prepulse alone trials $(74,78$, and $82 \mathrm{~dB}$ lasting $20 \mathrm{~ms}$ ), and three PPI trials where a $120 \mathrm{~dB}$ burst followed $100 \mathrm{~ms}$ after either of the three prepulse bursts. Background noise inside the chamber was set at $70 \mathrm{~dB}$. Startle responses were recorded for $65 \mathrm{~ms}$ following the onset of the pulse (or absence of). A randomly generated interval of $10-20 \mathrm{~s}$ was inserted between each trial.

\section{Statistical analyses}

Male and female behavioral data showed no significant sex-based differences, and therefore were combined in all statistical analyses. For all behavioral data, one-way analysis of variance was performed to compare all five groups based on the level of FMRP expression followed by Tukey's post hoc test. Western blotting and correlational analysis of FMRP transgene levels in different brain regions were compared with each mouse's behavioral data measured from the above-mentioned tests. GraphPad Prism v5 was used to perform the statistical analyses; a $p$-value of $<0.05$ was deemed statistically significant.

\section{RESULTS}

\section{AAV vectors and treatment groups}

Three different AAV9 vectors were studied in both WT and Fmr1 KO mice (Fig. 1). Two of the vectors contained the coding region of the mouse Fmr1 gene, but each carried a different promotor. The Syn-FMRP vector incorporated the human synapsin gene promoter previously shown to confer neuron-specific expression. ${ }^{21}$ The Syn-mCMVFMRP vector utilized a modified hybrid promoter consisting of the human synapsin 1 gene promoter in tandem with a minimal CMV enhancer element. ${ }^{24} \mathrm{~A}$ third vector ("null vector") containing a CMV promoter sequence, but no transgene served as a negative control. Five treatment groups were studied: (1) WT mice injected with the null vector served as the baseline controls; (2) WT mice injected with the Syn-FMRP vector; (3) Fmr1 KO mice injected with the null vector; (4) KO mice injected with the Syn-FMRP vector; and (5) KO mice injected with the Syn-mCMV-FMRP vector. Four to eight litters of newborn mice were used in each of the five treatment groups. All mice were injected under blinded conditions.

\section{FMRP transgene expression levels in adult mice after vector administration in neonates}

Quantitative Western blotting using an antiFMRP antibody was used to determine CNS transgene expression levels in young adult (2-3 months old) mouse brains. Five brain regions from mice in each treatment group were dissected and analyzed by Western blotting (Fig. 2A). The average FMRP expression levels were quantified and presented relative to the level in the WT null group. Examples of Western blots of the cortex, frontal cortex, and hippocampus of WT mice injected with the null vector or the Syn-FMRP (WT + FMRP) are compared with Fmr1 KO mice injected with the Syn-FMRP vector $(\mathrm{KO}+\mathrm{FMRP})$ and the Syn-mCMV-FMRP vector (KO + FMRP over) and are illustrated in Fig. 2B. Compared with WT null mice, an average \pm SEM level of FMRP expression was observed in Fmr1 KO mice injected with Syn-FMRP of $54 \pm 7 \%$ in the cerebral cortex, $115 \pm 18 \%$ in the frontal cortex, $80 \pm 15 \%$ in the hippocampus, $34 \pm 8 \%$ in the striatum, and $2 \pm 1 \%$ in the cerebellum (Fig. 2C). In WT mice injected with Syn-FMRP (Fig. 2D), the average levels of Fmr1 isoform 1 expression were $235 \pm 47 \%$ in the cortex, $182 \pm 30 \%$ in the frontal cortex, $209 \pm 45 \%$ in the hippocampus, $113 \pm 24 \%$ in the striatum, and $91 \pm 4 \%$ in the cerebellum. Fmr1 KO mice injected with the Syn-mCMV-FMRP vector showed higher levels of transgene expression compared with $\mathrm{KO}$ mice injected with Syn-FMRP (Fig. 2E). Expression in mice injected with Syn-mCMV-FMRP was $265 \pm 64 \%$ in the cortex, $446 \pm 231 \%$ in the frontal cortex, $255 \pm 41 \%$ in the hippocampus, $307 \pm 125 \%$ in the striatum, and $44 \pm 12 \%$ in the cerebellum compared with WT null mice.

\section{Distribution of the FMRP transgene}

Immunocytochemical analyses were carried out to document the CNS distribution of AAV vector expression in 2- to 3-month-old adult mice. Compared with a previous study where the Syn-FMRP vector was injected i.c.v. in Fmr1 KO mice at PND $5,{ }^{21}$ a wider distribution following i.c.v. injection of the FMRP transgene was achieved in the present study using a lower titer of the same vector administered prior to PND 3 (Fig. 3A). The Syn-FMRP 


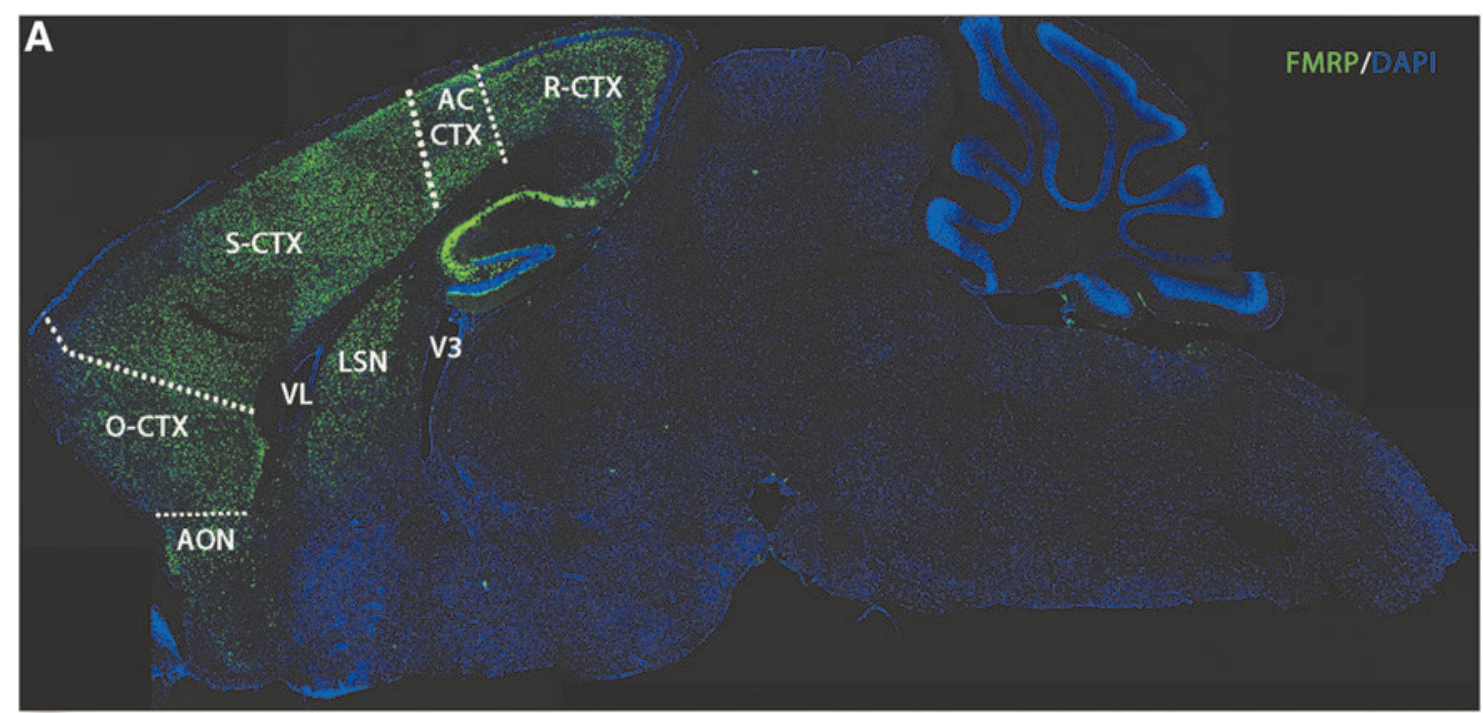

\begin{tabular}{|c|c|c|c|c|c|c|c|c|c|}
\hline Vector & 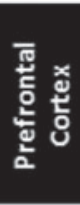 & 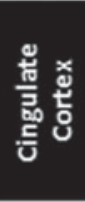 & 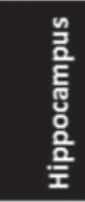 & 悬 & 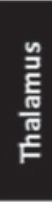 & 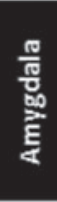 & 흘 & 흡 & 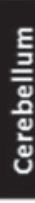 \\
\hline AAV-Syn-FMRP & +++ & +++ & +++ & n++ & ++ & ++ & ++ & + & + \\
\hline AAV-SynmCMV-FMRP & +++ & +++ & ++++ & ++ & ++ & ++ & ++ & ++ & + \\
\hline
\end{tabular}
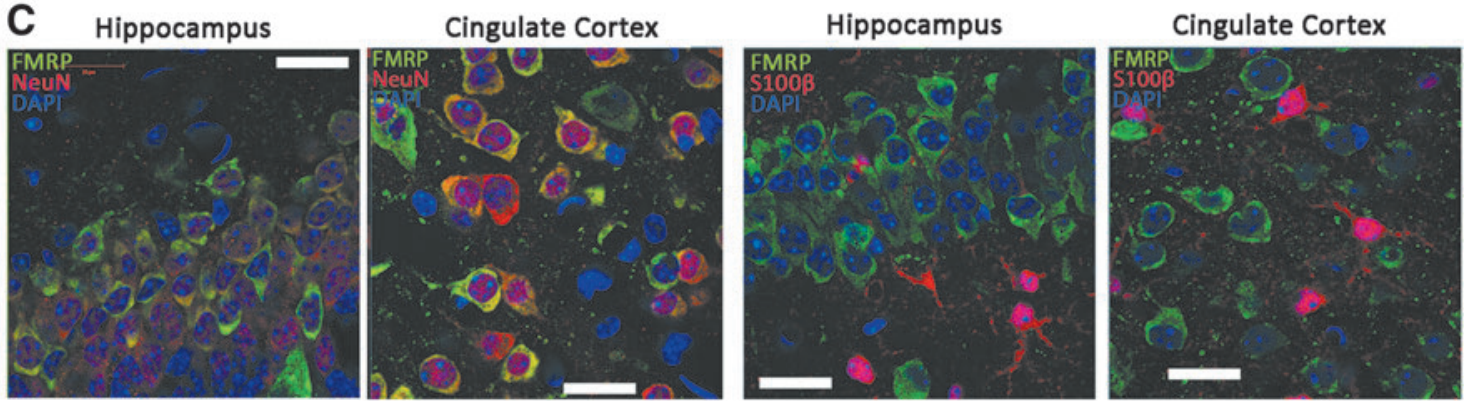

Figure 3. Immunocytochemical analysis of FMRP transgene distribution in the mouse CNS. (A) Low magnification image of a sagittal section showing FMRP transgene expression in a 2-month-old Fmr1 KO mouse injected with Syn-FMRP on postnatal day 1. FMRP is shown in green and DAPI in blue. R-CTX, retrosplenial cortex; Ac-CTX, anterior cingulate cortex; S-CTX, somatosensory cortex; 0-CTX, orbitofrontal cortex; AON: anterior olfactory nucleus; VL, lateral ventricle; LSN, lateral septal nucleus; V3, third ventricle. (B) Semi-quantitative assessment of the FMRP transgene distribution in 2-month-old Fmr1 KO mice. Brain regions were scored as (+) where 0-100 cells were counted, $(++)$ for 100-200 cells, (+++) for 200-300 cells, and (++++) for regions where $>300$ labelled cells were counted per $\mathrm{mm}^{2}$. No discernable differences were observed in the distribution or cell type tropism between the Syn-FMRP and Syn-mCMV-FMRP vectors. (C) Higher magnification photomicrographs of coronal sections from 2-month-old Fmr1 K0 mice injected with Syn-mCMV-FMRP showing co-labeling of NeuN (left panels) and S100 $\beta$ (right panels) in the cingulate cortex and hippocampus (CA1) brain regions (FMRP, green; NeuN or S100 $\beta$, red; nuclei counterstained with DAPI, blue). Scale bar $=20 \mu \mathrm{m}$.

transgene was detected in the anterior forebrain regions such as the orbitofrontal cortex and the anterior olfactory nucleus, which were not transduced in PND 5-treated animals. Also, in contrast to PND 5-injected neonates, FMRP was detected in adult Fmr1 KO mice injected with Syn-FMRP on PND 0-2 in other regions more distal from the injection site, including the amygdala, inferior colliculus, thalamus, and a few sporadic cells in the cerebellum (visible under higher magnification; data not shown). Similar results were observed in mice injected with the Syn-mCMV-FMRP vector (data not shown).

Although FMRP expression, as determined by Western blots, was substantially higher in Fmr1 KO mice injected with Syn-mCMV-FMRP vector (Fig. 2E) compared with Fmr1 KO mice injected with Syn-FMRP (Fig. 2C), the overall distribution of the two vectors in the CNS administered at comparable titers was very similar (Fig. 3B). In the 
cerebellum, very low levels of the transgene were detected with both vectors where a few FMRP positive Purkinje neurons were observed. A semiquantitative analysis of these brain regions showed that the most highly transduced regions were the cortex and hippocampus where $>200$ cells $/ \mathrm{mm}^{2}$ where transduced, while regions more distal to the lateral ventricles such as the thalamus and inferior colliculus were still transduced but to a lesser degree (Fig. 3B). WT animals injected with SynFMRP had a similar distribution of the transgene to that observed in KO-injected mice, but showed increased FMRP immunostaining in forebrain regions, including the cingulate cortex, striatum, and hippocampus (Supplementary Fig. S1; Supplementary Data are available online at www.liebertpub .com/hum).

Double labeling analyses using anti-NeuN and anti-S100 $\beta$ antibodies were carried out to assess neuronal versus glial tropism, respectively. Based on FMRP transgene co-localization with the neuronal marker NeuN, and as previously reported, ${ }^{9,21}$ the synapsin 1 promoter incorporated into the SynFMRP vector endowed neuron-specific tropism of FMRP in the CNS. Examples of coronal images from 2-month-old Fmr1 KO mice injected with SynmCMV-FMRP are illustrated in Fig. 3C. Despite the overall higher FMRP expression using this vector, the cellular tropism specific toward neurons was virtually identical to that seen with the lower expressing Syn-FMRP AAV vector. The cingulate cortex was selected for a full quantitative analysis of FMRP transgene cellular tropism with the two AAV vectors. Approximately $96 \%$ of NeuN-positive neurons in the cingulate cortex were transduced with Syn-mCMV-FMRP and 97\% with Syn-FMRP. Extensive NeuN positive co-localization with FMRP indicated that 94-96\% of the cells transduced with either vector were neurons (Supplementary Table S1). Thus, neuron-specific expression of FMRP using either Syn-FMRP or Syn-mCMV-FMRP is consistent with the native endogenous FMRP expression, which is predominantly neuronal in the adult mouse brain. ${ }^{11}$ Therefore, the main difference between the two FMRP vectors was the amount of FMRP expressed per neuron transduced.

In summary, a wider CNS distribution of the FMRP transgene was achieved following PND 0-2 bilateral i.c.v. administration compared with PND 5 injection, and cellular expression using both the Syn-FMRP and Syn-mCMV-FMRP vectors was nearly exclusively in neurons. Possible neuroinflammation induced by AAV vector injections into the lateral ventricles was also investigated. Glial fibrillary acidic protein (GFAP) was used as a marker to assess the status of astrocytes in a subset of injected mice. No signs of elevated GFAP immunostaining were seen near the ventricle or in nearby regions, including the striatum and hippocampus, indicating the absence of astrocytosis following bilateral i.c.v. administration of AAV vectors (data not shown).

\section{PSD-95 and MeCP2 in AAV-treated mice}

Taking advantage of (a) the modest transgene expression level achieved with the Syn-FMRP vector and higher levels obtained using the SynmCMV-FMRP vector, and (b) mouse-to-mouse variability in viral vector CNS transduction, this study sought to investigate the effects of inducing a range of FMRP transgene expression levels that would both translate into biochemical and behavioral correction in Fmr1 KO mice, and delineate the lower and upper limits of expression in terms of phenotypic rescue versus induction of pathology.

PSD-95 is a synaptic adapter and transducer protein that is highly expressed throughout the CNS. Previous work ${ }^{29}$ showing that PSD-95 mRNA is a substrate for FMRP by conducting immunoprecipitation of FMRP from WT forebrain followed by quantitative RT-PCR for PSD-95 mRNA was confirmed (Fig. 4A). Gapdh mRNA was used to normalize the total amount of mRNA; Gapdh protein and mRNA levels did not show any significant changes in WT compared to Fmr1 KO animals (data not shown). Previous studies also reported reduced PSD-95 protein in the Fmr1 KO mouse brain. ${ }^{29,30}$ Therefore, the ability of FMRP transgene expression to normalize PSD-95 levels in adult $\mathrm{KO}$ mouse brain following neonatal administration of AAV vectors was examined. PSD-95 protein in the cerebral cortex of $\mathrm{KO}$ null vectorinjected mice was reduced by $28 \pm 7 \%$ compared with that observed in the WT null group (Fig. 4B). In the Syn-FMRP-treated Fmr 1 KO group, PSD-95 levels were restored to the level seen in the WT null mice. No significant changes in PSD-95 protein were observed in WT mice injected with Syn-FMRP or in Fmr1 KO mice injected with Syn-mCMVFMRP compared to the WT null group, indicating that FMRP over-expression above native WT levels had little impact on PSD-95 protein levels.

The effects of FMRP expression on the transcription regulator MeCP2 were also investigated. Mutations in the MECP2 gene cause downregulation of MeCP2 protein and induce Rett syndrome, another neurodevelopmental disorder associated with autistic symptoms. MeCP2 mRNA was previously listed as a potential substrate for FMRP, ${ }^{7}$ and MeCP2 mRNA contains G-quadruplex 
A

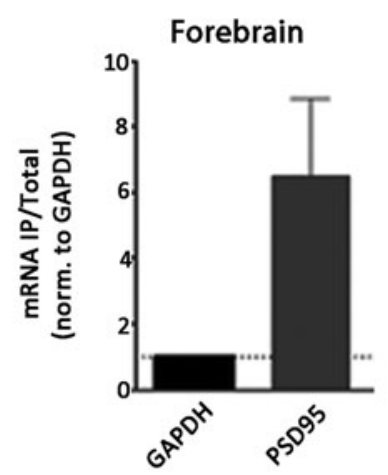

C

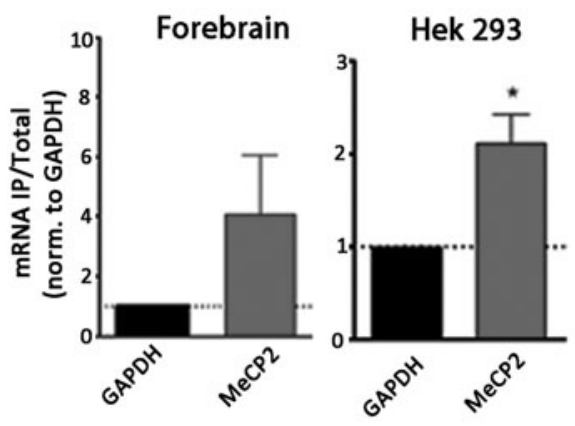

B

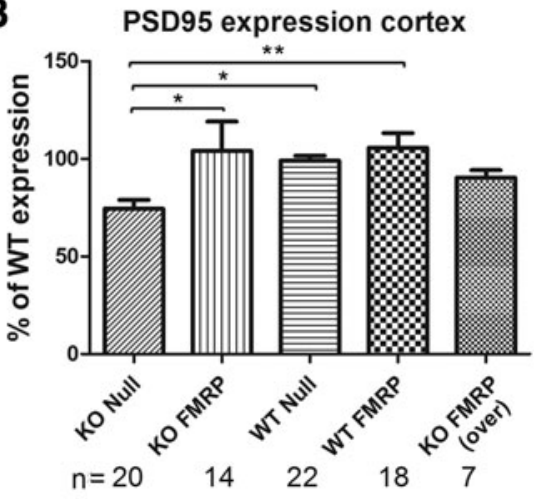

D

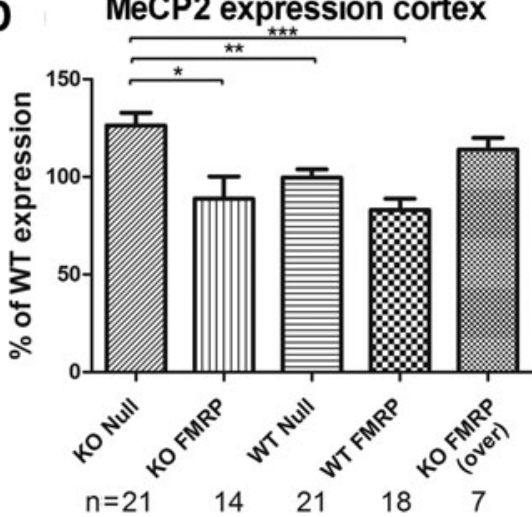

Figure 4. Characterization of PSD-95 and MeCP2 in Fmr1 KO and WT mice. (A) FMRP immunoprecipitation and mRNA quantification of total mRNA enrichment of PSD-95 from WT brain. (B) PSD-95 protein levels in the cortex from the five treatment groups relative to the expression in WT null mice. (C) FMRP immunoprecipitation and mRNA quantification of total MeCP2 mRNA enrichment from whole WT forebrain and HEK-293 cells. (D) Relative expression of MeCP2 protein in the cortex. Results in (A) and (C) were normalized to GAPDH mRNA levels, and those in (B) and (D) were corrected based on GAPDH protein. Results represent means \pm SEM. ${ }^{*} p<0.05 ;{ }^{* *} p<0.01 ;{ }^{* * *} p<0.001$.

sites, ${ }^{31}$ a motif present in many FMRP mRNA substrates. ${ }^{7,32}$ To establish whether MeCP2 mRNA is indeed a bona fide FMRP substrate, additional FMRP immuno-precipitation/RT-PCR experiments were conducted to determine if MeCP2 mRNA was enriched in the FMRP immuno-precipitate. An enrichment of MeCP2 mRNA was seen in whole forebrain isolates from WT mice (Fig. 4C). An enrichment of MeCP2 mRNA following FMRP immunoprecipitation was also seen in human embryonic kidney-293 cells, which endogenously express FMRP and MeCP2 (Fig. 4C). Thus, both Homo sapiens and Mus musculus MeCP2 mRNA interacts with FMRP. To ascertain the functional relevance of this finding, $\mathrm{MeCP} 2$ protein expression levels in the cerebral cortex of AAV vector-treated mice were quantified. In contrast to reduced PSD-95 in null vector-treated Fmr1 KO mice, MeCP2 protein expression was elevated by $26 \pm 6 \%$ in the cortex compared with WT null levels (Fig. 4D). Importantly, the reintroduction of FMRP normalized MeCP2 expression in Fmr1 KO mice, while FMRP over-expression in WT and $\mathrm{KO}$ mice had little effect on MeCP2 protein (Fig. 4D).
These results demonstrate that the introduction of FMRP via AAV vectors recapitulates the native translational modulation normally conferred by endogenous FMRP.

\section{Effects of viral vector-mediated expression of FMRP on behavior}

Motor activity. Behavioral testing was initiated by assessing whether the well-documented motor hyperactivity in Fmr1 KO mice ${ }^{30,33,34}$ was affected by administration of FMRP AAV vectors. Locomotor activity parameters did not correlate with FMRP expression levels over the entire range of all FMRP positive groups. However, as expected, the KO null group displayed significantly increased horizontal (Fig. 5A) and ambulatory activity (Fig. 5B), and nonsignificant reductions in rest time (Fig. 5C) and rest episodes (Fig. 5D) compared with the WT null group. The Fmr $1 \mathrm{KO}$ group treated with Syn-FMRP showed reduced horizontal and ambulatory activities and a non-significant elevation of rest time and episodes compared with the null-injected $\mathrm{KO}$ con- 

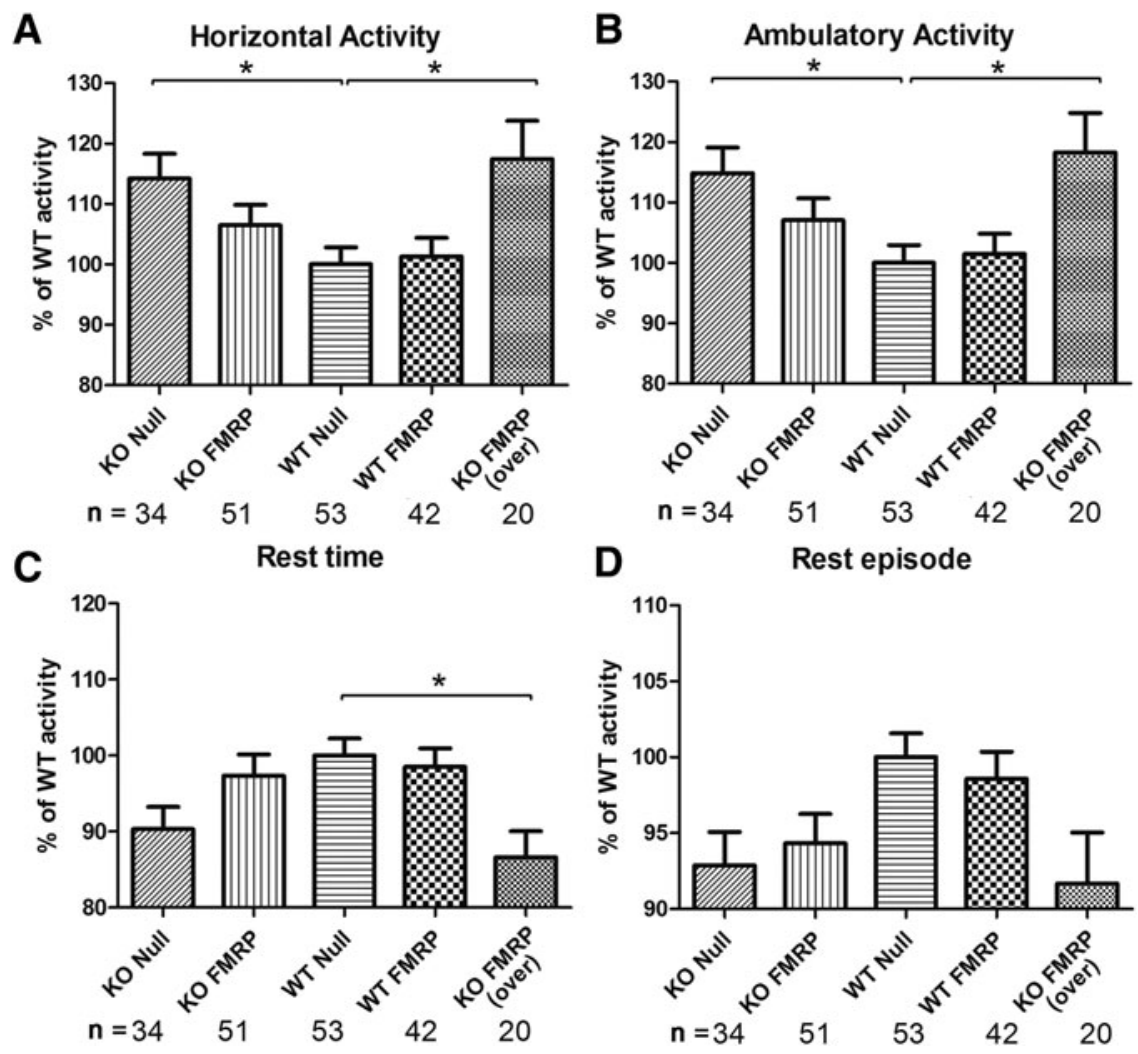

Figure 5. Analysis of locomotor activity. (A) Horizontal activity for KO null, KO + FMRP, WT null, WT + FMRP, and KO + FMRP (over) groups during locomotor testing. (B) Ambulatory activity. (C) Rest episodes as measured by the number of pauses (absence of beam breaks for $\geq 1 \mathrm{~s}$ ). (D) Rest episodes (number of pauses in motion). Horizontal activity encompasses all incidences of motion in the horizontal field (ambulation + grooming + stereotypies + rearing), whereas ambulatory activity counts motion only when the center of mass is displaced. Results are shown normalized to WT null activity and are presented as means \pm SEM. ${ }^{*} p<0.05$.

trols, indicating a partial rescue of some hyperactive behavioral parameters. Modest over-expression in the WT + FMRP injected with Syn-FMRP showed no effects on motor activity compared to null-injected WT mice (Fig. 5A-D). In contrast, higher overexpression of FMRP in the Fmr1 KO mice injected with the Syn-mCMV-FMRP vector resulted in elevated horizontal and ambulatory activity (Fig. 5A and $\mathrm{B}$ ) and reduced rest time (Fig. 5C) compared with the WT null mice. Thus, excessively high overexpression of FMRP in the Fmr $1 \mathrm{KO}$ mouse forebrain appears to mimic the hyperactive endophenotype observed in the null vector-treated Fmr1 KO mouse with no FMRP.

Elevated plus maze. In the elevated plus maze measuring anxiety, the Fmr1 KO null group showed significantly more time in the open arms and more entries into the open arms compared with the WT null group (Fig. 6A and C). This result is consistent with a previous report on Fmr1 mice showing an apparent reduction in anxiety. ${ }^{35}$ The abnormal elevation in open-arm time in the $\mathrm{KO}$ null mice was fully corrected in the $\mathrm{KO}+\mathrm{FMRP}$ treatment group (Fig. 6A). The elevated open-arm entries in the $\mathrm{KO}$ null group were reduced in the $\mathrm{KO}+$ FMRP group treated with Syn-FMRP, although they was still elevated compared with the WT null mice, suggesting a partial rescue (Fig. 6C). The closed-arm entries of the KO + FMRP treatment group were significantly higher compared with the KO null group, but not different from the WT null controls, again suggesting rescue based on this parameter (Fig. 6E). The total number of entries was not different between groups, indicating that locomotor hyperactivity was not a driving factor in this test (data not shown). Notably, correlational analyses indicated a significant correlation between FMRP levels in the striatum and the total time spent in the open arms (Fig. 6B; $R=$ $-0.38, p<0.02, n=40$ ) as well as striatal FMRP with the total number of open-arm entries (Fig. 6D; $R=-0.37, p<0.02, n=40$ ). Except for the number of open-arm entries where the WT + FMRP group showed an elevation compared with WT null mice (Fig. 6C), modest over-expression in WT mice with 
A
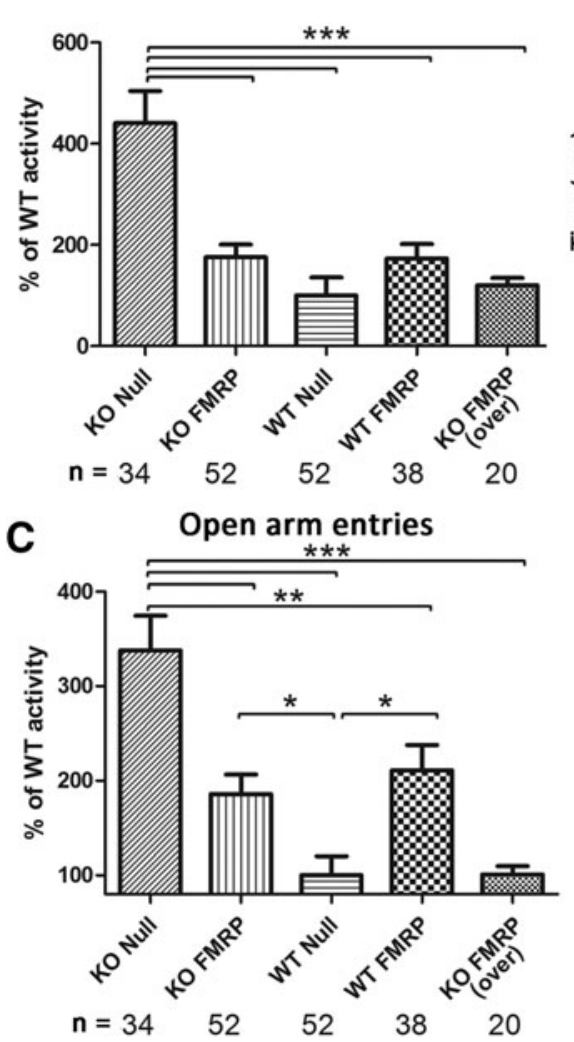

E

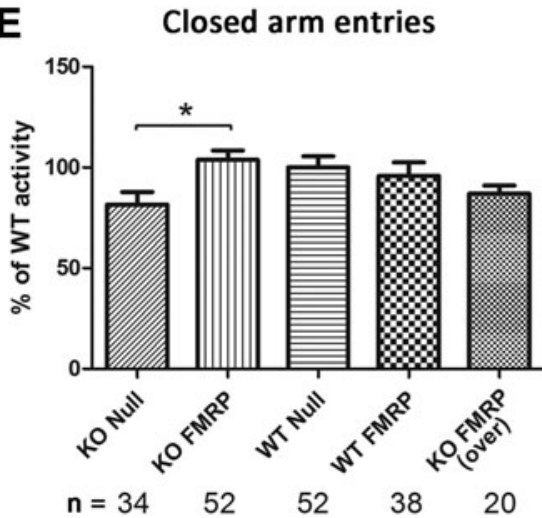

B

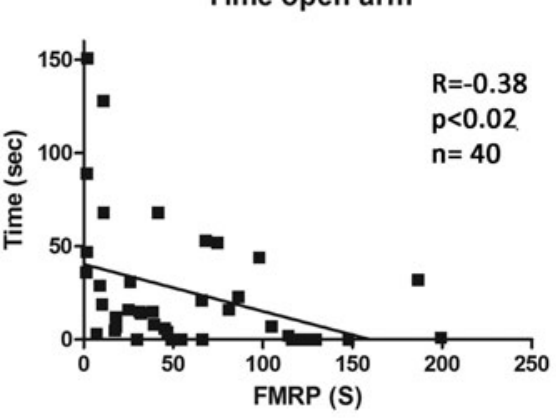

D Open arm entries

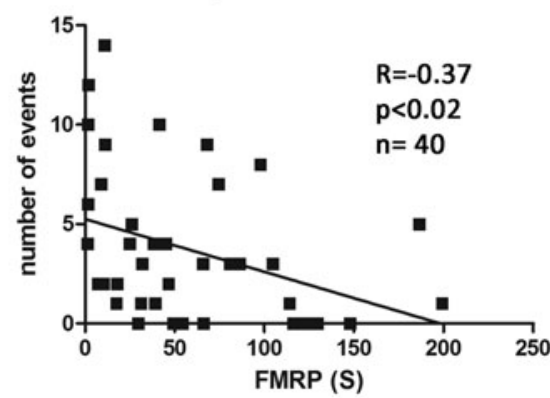

$\mathbf{F}$

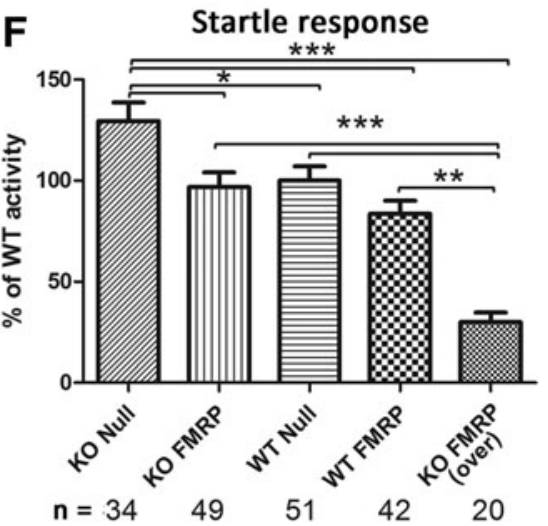

Figure 6. Results of the elevated plus maze and startle response. (A) Total time spent in the open arms for the K0 null, KO + FMRP, WT null, WT + FMRP, and $\mathrm{KO}+$ FMRP (over) groups, and (C) number of open-arm and (E) number of closed-arm entries. Panels (B) and (D) indicate correlational analyses between FMRP transgene levels in the striatum based on Western blot analysis and the time in the open arms (B) and open-arm entries (D). (F) Basal startle response obtained from a $120 \mathrm{~dB}$ pulse for the KO null, KO + FMRP, WT null, WT + FMRP, and KO + FMRP (over) groups. Results are normalized relative to WT null activity and presented as means \pm SEM. ${ }^{*} p<0.05 ;{ }^{* *} p<0.01 ;{ }^{* * *} p<0.001$.

the Syn-FMRP vector, or high over-expression in the KO mice injected with the Syn-mCMV-FMRP vector, had little or no effect on non-social anxiety (Fig. 6A and E).

\section{Analysis of sensorimotor gating}

Impaired sensorimotor gating and auditory hyperexcitability have been shown to be robust biomarkers in Fmr1 KO mice. ${ }^{27}$ Therefore, pre-pulse inhibition and the base startle responses were investigated. Pre-pulse inhibition at 74,78 , and $82 \mathrm{~dB}$ was not different between the WT and $\mathrm{KO}$ null-injected groups (data not shown). However, the KO null group had a significantly higher base startle response than the WT null group had. This difference was completely neutralized in the $\mathrm{KO}+\mathrm{FMRP}$ treatment group, demonstrating correction of this abnormality (Fig. 6F). Modest over-expression in the WT + FMRP group showed a non-significant reduction in the startle response, while the high over-expression $\mathrm{KO}$ group displayed 
a larger and significant reduction in startle response compared with the baseline WT null group (Fig. 6F). Therefore, on this behavioral parameter, there appeared to be a dose-response effect whereby elevated startle in the Fmr $1 \mathrm{KO}$ group was corrected by administration of the FMRP transgene, and increasing FMRP above WT levels further suppressed the acoustic startle response.

\section{DISCUSSION}

To study the efficacy of AAV FMRP viral vectors in the CNS, AAV serotype 9-based vectors that are capable of efficiently transducing brain parenchyma were employed. ${ }^{36}$ The use of the synapsin promoter permitted long-term neuron-specific tropism of the FMRP transgene protein. This is important because FMRP is expressed predominantly in neurons throughout all regions of the adult mouse CNS. ${ }^{11}$ Previous work demonstrated that AAVmediated FMRP transgene expression in the brain remains relatively constant in terminally differentiated neurons for at least 7 months after i.c.v. administration. ${ }^{21}$ In the present study, bilateral i.c.v. injection into mice on PND 0-2 provided superior diffusion of the vector within the forebrain compared with results obtained previously when the same vector (Syn-FMRP) was administered i.c.v. on PND $5 .^{21}$ The superiority of immediate postnatal i.c.v. AAV treatment was also illustrated in a gene therapy study using a mouse model of TSC, another disorder on the autism spectrum. An AAV vector coding for TSC1 injected into the ventricles at PND 0 resulted in widespread diffusion of the vector, and in this case, therapeutic efficacy was demonstrated by increased survival time compared with vehicle-injected controls. ${ }^{19}$ The ependymal lining surrounding the ventricles of the brain is still immature within the first 2 days after birth, and therefore AAV vectors can more easily diffuse to more distal regions within the brain parenchyma when injected at this early time point compared with injections made only a few days later. ${ }^{10,37,38}$

The experimental design used here employing three different AAV vectors and five different treatment groups provided an opportunity to examine a wide range of FMRP expression levels in the brain. These extended from no FMRP expression in the Fmr1 KO null group to intermediate expression in the KO Syn-FMRP group, normal expression in the WT null group (designated as $100 \%$ of baseline), modest over-expression in WT mice treated with the Syn-FMRP vector, and, on the upper end, excessive over-expression in the
Fmr1 KO using the Syn-mCMV-FMRP vector. This experimental arrangement allowed the full spectrum of effects induced by variable levels of FMRP forebrain expression to be delineated.

FMRP is an mRNA binding and trafficking protein that regulates the localization and expression of hundreds of genes. ${ }^{7,8}$ In its absence, as in FXS, the levels of expression of proteins whose mRNAs are bound and regulated by FMRP, can be either up- or downregulated depending, in part, on the stability of the mRNA substrate. PSD-95 is a highly expressed protein crucial for synaptic integrity and plasticity. PSD-95 mRNA is an established substrate of FMRP and PSD-95 protein is downregulated in the absence of FMRP. ${ }^{29,30}$ It was found that Fmr1 KO mice treated with SynFMRP displayed complete restoration of PSD95 protein in the cerebral cortex. Thus, FMRP transgene-mediated correction of pathologically low PSD-95 demonstrates recapitulation of the normal translational control function of FMRP in neurons.

MeCP2 mRNA was also identified as a substrate for FMRP. This X-linked MeCP2 gene is mutated in Rett syndrome, another neurodevelopmental disorder associated with autistic features. MeCP2 functions as a transcriptional modulator of a wide array of genes, ${ }^{39,40}$ and appears to be particularly important in the regulation of long genes $(>100 \mathrm{~Kb})$ in the brain. ${ }^{41}$ Unlike PSD-95, which was reduced, levels of MeCP2 protein were elevated in null-treated Fmr1 KO mouse brains. The absence of changes in the expression profile of MeCP2 and PSD-95 protein in WT mice treated with the Syn-FMRP vector (modest over-expression) or in Fmr1 KO mice treated with the high expressing Syn-mCMV-FMRP vector indicates that the effect of FMRP on the translation on these two substrates does not follow a linear doseresponse profile once brain FMRP levels rise above normal WT levels. Elevated MeCP2 in the Fmr1 KO brain is consistent with FMRP acting as a translation inhibitor in WT mice. More importantly, abnormally increased MeCP2 expression in the Fmr1 KO CNS suggests the possibility that increased MeCP2 might play a role in the overall FXS phenotype. MeCP2 over-expressing mice display impaired synaptic plasticity and autistic behaviors, ${ }^{42,43}$ and like both FXS and FMR1 gene duplication syndrome, duplication of the human MeCP2 gene is characterized by developmental delay, mental retardation, and seizures. ${ }^{44-47}$ The overlapping symptomatic features of FXS, Rett syndrome, and FMR1 and MeCP2 gene duplication syndromes suggests that further studies investigating the relationships of these two key regulatory proteins is warranted. 
Demonstrating the correction of abnormal behaviors is an essential requirement for studies seeking to develop treatments for FXS and other autism spectrum disorders. Several behaviors in the Fmr1 KO mouse were investigated that are known to be well-established endophenotypes in Fmr1 KO mice: motor hyperactivity, startle response for assessing sensorimotor gating, and analysis of non-social anxiety in the elevated plus maze (summarized in Table 1). Motor hyperactivity and abnormal behaviors in the elevated plus maze and the startle response in the null-treated Fmr1 KO mice were significantly altered compared with null-treated WT mice, and these abnormal behaviors were fully or partially rescued after moderate FMRP expression.

Curiously, in the elevated plus maze, the Fmr1 KO null group displayed a highly significant increase in the number of entries and the time spent in the open arms and decreased time in the closed arms, indicative of reduced anxiety. Reduced anxious behavior in the elevated plus maze and other tests of non-social anxiety have previously been reported for the Fmr1 KO mouse. ${ }^{35,48,49}$ This phenomenon has also been observed in other mutant mouse lines. For example, $\mu$ opioid receptor KO mice, which display a general "autistic profile" on a battery of behavioral tests and have been proposed as a novel animal model of autism, ${ }^{50}$ show reduced anxiety behavior in the elevated plus maze. ${ }^{51,52}$ Although the elevated plus maze test is traditionally viewed as an assessment of anxiety, in the context of an overall "autistic phenotype," the test may additionally or instead reflect hyper-arousal to novel environments and/or reduced cognitive function in conjunction with reduced fear-that is, the mice may be less cognizant of the potential danger of open spaces (e.g., open arms in the elevated plus maze).
A primary objective of this study was to provide insight into the upper and lower limits of FMRP expression in the Fmr1 mouse brain that rectify abnormal biochemical and behavioral parameters without inducing harmful effects from overexpression. Over-expression of FMRP likely has deleterious consequences, as persons carrying an FMR1 gene duplication show profound physical changes and cognitive impairment. Several cases of this syndrome have been reported with common features that include severe mental retardation, developmental delay, and seizures. ${ }^{53-56}$ Transgenic mice massively over-expressing human FMRP at 10- to 15-fold above normal WT levels of the mouse gene showed increased anxiety and reduced motor activity. ${ }^{48}$ These results differ from the current results where Fmr1 KO mice treated with the high expressing Syn-mCMV-FMRP vector showed no major changes in anxiety behavior and a robust increase in motor activity. Possible explanations for this discrepancy include (a) the large differences in protein expression levels (10- to 15 -fold above WT in Peier et al., and 2.5- to 4.5-fold in the present study); (b) differences in the gene promotors used: (presumably) the endogenous human FMR1 promotor in the transgenic mice versus the synapsin/minimal CMV promoter used here that directed expression solely to neurons; and (c) body-wide over-expression in the transgenic mice versus FMRP transgene expression restricted to forebrain regions in the present study.

Two additional important findings can be gleaned from this study. First, both biochemical rescue (as determined by measuring FMRP substrates) and behavioral rescue were apparent in Fmr1 KO mice expressing FMRP in the forebrain at levels moderately below and above the WT level. Second, low or modest over-expression of FMRP did not appear to induce pathology. The former point is

Table 1. Summary of behavior in treated mice

\begin{tabular}{|c|c|c|c|c|c|c|}
\hline Test & Behavior & \multicolumn{5}{|c|}{ Treatment groups } \\
\hline Locomotor activity & Ambulation & Normal & $\uparrow$ & PR & NC & $\uparrow$ \\
\hline Sensorimotor gating & Startle response & Normal & $\uparrow$ & $\nu$ & NC & $\downarrow \downarrow$ \\
\hline \multirow[t]{2}{*}{ Elevated plus maze } & Non-social anxiety & Normal & $\downarrow$ & $\boldsymbol{v}$ & $\mathrm{NC}$ & $\nu$ \\
\hline & Avoidance & Normal & $\downarrow$ & $\boldsymbol{\nu}$ & $\mathrm{NC}$ & $\mathrm{NC}$ \\
\hline
\end{tabular}

The locomotor, sensorimotor, and non-social anxiety-like phenotypes of the five treatment groups are indicated. Ambulation includes horizontal and ambulatory activities, while rest encompasses rest time and rest episodes. Avoidance is defined as the number of entries into the closed arm, and exploration is the number of entries into the open arm of the elevated plus maze.

WT, wild type; KO, knockout; FMRP, fragile X mental retardation protein; $\uparrow$ and $\downarrow$, the directionality of the pathological deviation from the normal WT null activity $(p<0.05) ; \boldsymbol{V}$, tests where pathological behavior was fully rescued $(p<0.05)$; PR, partial correction; NC, no significant change compared to WT null activity $(p>0.05)$. 
based on normalization of FMRP substrates and reversal of behavioral abnormalities in $\mathrm{KO}$ mice expressing the FMRP transgene in the forebrain at approximately $35-115 \%$ relative to WT mice, depending on the brain region. The latter suggestion is based on the absence of effects on FMRP substrates (PSD-95 and MeCP2) and little or no effects on behavior in WT mice expressing FMRP at 115$235 \%$ above WT levels. Taken together, these results indicate that a certain degree of leeway exists in terms of the vector-mediated transgene expression levels required in CNS neurons for achieving phenotypic reversal without inducing deleterious effects. In light of the various dosing and pharmacokinetic issues associated viral vectorbased therapeutics noted above, these findings provide encouraging support for the development of AAV-FMRP as a viable human biopharmaceutical therapeutic agent.

\section{ACKNOWLEDGMENTS}

We thank Drs. James Eubanks and Lu-Yang Wang for helpful comments on the manuscript. This study was supported by the Canadian Institute of Health Research and the Fragile X Research Foundation of Canada. J.A. is a recipient of a Postdoctoral Fellowship from the Fragile X Research Foundation of Canada.

\section{AUTHOR DISCLOSURE}

No competing financial interests exist.

\section{REFERENCES}

1. Jacquemont $S$, Berry-Kravis $E$, Hagerman $R$, et al. The challenges of clinical trials in Fragile $X$ Syndrome. Psychopharmacology 2014;231:1237-1250.

2. Hampson DR, Gholizadeh S, Pacey LK. Pathways to drug development for autism spectrum disorders. Clin Pharmacol Ther 2012;91:189-200.

3. Ebrahimi-Fakhari D, Sahin M. Autism and the synapse: emerging mechanisms and mechanismbased therapies. Curr Opin Neurol 2015;28:91102.

4. Wang H, Pati S, Pozzo-Miller L, et al. Targeted pharmacological treatment of autism spectrum disorders: Fragile $\mathrm{X}$ and Rett syndromes. Front Cell Neurosci 2015;9:55.

5. Berry-Kravis E. Mechanism-based treatments in neurodevelopmental disorders: Fragile X Syndrome. Pediatr Neurol 2014;50:297-302.

6. Schaefer TL, Davenport MH, Erickson CA. Emerging pharmacologic treatment options for Fragile X Syndrome. Appl Clin Genet 2015;8:75-93.

7. Darnell JC, Van Driesche SJ, Zhang C, et al. FMRP stalls ribosomal translocation on mRNAs linked to synaptic function and autism. Cell 2011;146:247261.

8. Ascano M Jr, Mukherjee N, Bandaru P, et al. FMRP targets distinct mRNA sequence elements to regulate protein expression. Nature 2012;492: 382-386.

9. Gholizadeh S, Tharmalingam S, Macaldaz ME, et al. Transduction of the central nervous system after intracerebroventricular injection of adenoassociated viral vectors in neonatal and juvenile mice. Hum Gene Ther Methods 2013;24:205-213.

10. Kim JY, Grunke SD, Levites Y, et al. Intracerebroventricular viral injection of the neonatal mouse brain for persistent and widespread neuronal transduction. J Vis Exp 2014;51863.
11. Gholizadeh S, Halder SK, Hampson DR. Expression of fragile $X$ mental retardation protein in neurons and glia of the developing and adult mouse brain. Brain Res 2015;1596:22-30.

12. Kantor B, Bailey RM, Wimberly K, et al. Methods for gene transfer to the central nervous system. Adv Genet 2014;87:125-197.

13. Samulski RJ, Muzyczka N. AAV-mediated gene therapy for research and therapeutic purposes. Ann Rev Virol 2014;1:427-451.

14. Naldini L. Gene therapy returns to centre stage. Nature 2015;526:351-360.

15. Daily JL, Nash K, Jinwal U, et al. Adenoassociated virus-mediated rescue of the cognitive defects in a mouse model for Angelman syndrome. Plos One 2011;6:e27221.

16. Gadalla KK, Bailey ME, Spike RC, et al. Improved survival and reduced phenotypic severity following AAV9/MECP2 gene transfer to neonatal and juvenile male Mecp2 knockout mice. Mol Ther 2013;21:18-30.

17. Garg SK, Lioy DT, Cheval H, et al. Systemic delivery of MeCP2 rescues behavioral and cellular deficits in female mouse models of Rett syndrome. J Neurosci 2013;33:13612-13620.

18. Katz DM, Bird A, Coenraads M, et al. Rett syndrome: crossing the threshold to clinical translation. Trends Neurosci 2016;39:100-113.

19. Prabhakar S, Zhang X, Goto J, et al. Survival benefit and phenotypic improvement by hamartin gene therapy in a tuberous sclerosis mouse brain model. Neurobiol Dis 2015;82:22-31.

20. Zeier Z, Kumar A, Bodhinathan K, et al. Fragile X mental retardation protein replacement restores hippocampal synaptic function in a mouse model of Fragile X Syndrome. Gene Ther 2009;16:11221129
21. Gholizadeh S, Arsenault J, Xuan IC, et al. Reduced phenotypic severity following adeno-associated virus-mediated FMR1 gene delivery in fragile $X$ mice. Neuropsychopharmacology 2014;39:31003111

22. Karda R, Buckley SM, Mattar CN, et al. Perinatal systemic gene delivery using adeno-associated viral vectors. Front Mol Neurosci 2014;7:89.

23. Serguera C, Bemelmans AP. Gene therapy of the central nervous system: general considerations on viral vectors for gene transfer into the brain. Rev Neurol (Paris) 2014;170:727-738.

24. Matsuzaki $Y$, Oue M, Hirai $H$. Generation of a neurodegenerative disease mouse model using lentiviral vectors carrying an enhanced synapsin I promoter. J Neurosci Methods 2014;223:133143.

25. Gabel LA, Won S, Kawai H, et al. Visual experience regulates transient expression and dendritic localization of fragile $X$ mental retardation protein. J Neurosci 2004;24:10579-10583.

26. LaFauci G, Adayev T, Kascsak R, et al. Fragile X screening by quantification of FMRP in dried blood spots by a Luminex immunoassay. J Mol Diagn 2013;15:508-517.

27. Paylor R, Yuva-Paylor LA, Nelson DL, et al. Reversal of sensorimotor gating abnormalities in Fmr1 knockout mice carrying a human Fmr1 transgene. Behav Neurosci 2008;122:1371-1377.

28. Veeraragavan S, Graham D, Bui N, et al. Genetic reduction of muscarinic M4 receptor modulates analgesic response and acoustic startle response in a mouse model of Fragile $X$ Syndrome (FXS). Behav Brain Res 2012;228:1-8.

29. Zalfa F, Eleuteri B, Dickson KS, et al. A new function for the fragile $X$ mental retardation protein in regulation of PSD-95 mRNA stability. Nat Neurosci 2007;10:578-587. 
30. Pacey LK, Doss L, Cifelli C, et al. Genetic deletion of regulator of G-protein signaling 4 (RGS4) rescues a subset of fragile $X$ related phenotypes in the FMR1 knockout mouse. Mol Cell Neurosci 2011:46:563-572

31. Bagga JS, D'Antonio LA. Role of conserved cisregulatory elements in the post-transcriptional regulation of the human MECP2 gene involved in autism. Hum Genom 2013;7:19.

32. Wang T, Bray SM, Warren ST. New perspectives on the biology of Fragile $X$ Syndrome. Curr Opin Genet Devel 2012;22:256-263.

33. Restivo L, Ferrari F, Passino E, et al. Enriched environment promotes behavioral and morphological recovery in a mouse model for the Fragile $X$ Syndrome. Proc Natl Acad Sci U S A 2005; 102:11557-11562.

34. Yan QJ, Rammal M, Tranfaglia M, et al. Suppression of two major Fragile $X$ Syndrome mouse model phenotypes by the mGluR5 antagonist MPEP. Neuropharmacology 2005;49:1053-1066

35. Qin M, Xia Z, Huang T, et al. Effects of chronic immobilization stress on anxiety-like behavior and basolateral amygdala morphology in Fmr1 knockout mice. Neuroscience 2011:194:282-290.

36. McLean JR, Smith GA, Rocha EM et al. Widespread neuron-specific transgene expression in brain and spinal cord following synapsin promoterdriven AAV9 neonatal intracerebroventricular injection. Neurosci Lett 2014;576:73-78.

37. Passini MA, Wolfe JH. Widespread gene delivery and structure-specific patterns of expression in the brain after intraventricular injections of neonatal mice with an adeno-associated virus vector J Virol 2001;75:12382-12392.

38. Chakrabarty P, Rosario A, Cruz P, et al. Capsid serotype and timing of injection determines AAV transduction in the neonatal mice brain. PLOS ONE 2013;8:e67680.
39. Lyst MJ, Bird A. Rett syndrome: a complex disorder with simple roots. Nat Rev Genet 2015;16: 261-275.

40. Pohodich AE, Zoghbi HY. Rett syndrome: disruption of epigenetic control of postnatal neurological functions. Hum Mol Genet 2015;24:R10-R16.

41. Gabel HW, Kinde B, Stroud H, et al. Disruption of DNA-methylation-dependent long gene repression in Rett syndrome. Nature 2015;522:89-93.

42. Na ES, Nelson ED, Adachi $M$, et al. A mouse model for MeCP2 duplication syndrome: МeCP2 overexpression impairs learning and memory and synaptic transmission. J Neurosci 2012;32:31093117.

43. Sztainberg Y, Chen HM, Swann JW, et al. Reversal of phenotypes in MECP2 duplication mice using genetic rescue or antisense oligonucleotides. Nature 2015:528:123-126.

44. Smyk M, Obersztyn E, Nowakowska B, et al Different-sized duplications of Xq28, including MECP2 in three males with mental retardation, absent or delayed speech, and recurrent infections. Am J Med Genet B Neuropsychiatr Genet 2008;147B:799-806.

45. Ramocki MB, Tavyev YJ, Peters SU. The MECP2 duplication syndrome. Am J Med Genet A 2010; 152A:1079-1088.

46. Reardon W, Donoghue V, Murphy AM, et al. Progressive cerebellar degenerative changes in the severe mental retardation syndrome caused by duplication of MECP2 and adjacent loci on Xq28. Eur J Pediatr 2010;169:941-949.

47. Van Esch H. MECP2 duplication syndrome. Mol Syndromol 2012;2:128-136.

48. Peier AM, Mcllwain KL, Kenneson A, et al. (Over)correction of FMR1 deficiency with YAC transgenics: behavioral and physical features. Hum Mol Genet 2000;9:1145-1159.
49. Liu ZH, Smith CB. Dissociation of social and nonsocial anxiety in a mouse model of Fragile $X$ Syndrome. Neurosci Lett 2009;454:62-66.

50. Becker JA, Clesse D, Spiegelhalter C, et al Autistic-like syndrome in mu opioid receptor null mice is relieved by facilitated mGluR4 activity. Neuropsychopharmacol 2014;39:2049-2060.

51. Filliol D, Ghozland S, Chluba J, et al. Mice deficient for delta- and mu-opioid receptors exhibit opposing alterations of emotional responses. Nat Genet 2000;25:195-200.

52. Ide S, Sora I, Ikeda K, et al. Reduced emotiona and corticosterone responses to stress in muopioid receptor knockout mice. Neuropharmacology 2010;58:241-247.

53. Rio $\mathrm{M}$, Malan V, Boissel $\mathrm{S}$, et al. Familial interstitial Xq27.3q28 duplication encompassing the FMR1 gene but not the MECP2 gene causes a new syndromic mental retardation condition. Eur $\mathrm{J}$ Hum Genet 2010;18:285-290.

54. Nagamani SC, Erez A, Probst FJ, et al. Small genomic rearrangements involving FMR1 support the importance of its gene dosage for normal neurocognitive function. Neurogenetics 2012;13:333-339.

55. Vengoechea J, Parikh AS, Zhang S, et al. De novo microduplication of the FMR1 gene in a patient with developmental delay, epilepsy and hyperactivity. Eur J Hum Genet 2012;20:1197-1200.

56. Hickey SE, Walters-Sen L, Mosher TM, et al. Duplication of the Xq27.3-q28 region, including the FMR1 gene, in an X-linked hypogonadism, gynecomastia, intellectual disability, short stature, and obesity syndrome. Am J Med Genet A 2013; 161A:2294-2299.

Received for publication June 20, 2016 accepted after revision August 26, 2016

Published online: September 7, 2016 